\title{
Clinical significance of serum tumor markers for advanced gastric cancer with the first-line chemotherapy
}

\author{
Zhiwei Sun, Jun Jia, Feng Du, Ying Yang, Chuanling Liu, Yanjie Xiao, Jing Yu, Xiaodong Zhang \\ VIP-II Division of Medical Department, Key Laboratory of Carcinogenesis and Translational Research (Ministry of Education, Beijing), Peking \\ University Cancer Hospital and Institute, Beijing 100142, China \\ Contributions: (I) Conception and design: X Zhang; (II) Administrative support: X Zhang, J Jia; (III) Provision of study materials or patients: Z Sun; (IV) \\ Collection and assembly of data: F Du, C Liu, J Yu, Y Yang, Y Xiao; (V) Data analysis and interpretation: Z Sun, J Jia; (VI) Manuscript writing: All \\ authors; (VII) Final approval of manuscript: All authors. \\ Correspondence to: Dr. Xiaodong Zhang. VIP-II Division of Medical Department, Key Laboratory of Carcinogenesis and Translational Research \\ (Ministry of Education, Beijing), Peking University Cancer Hospital and Institute, 52 Fucheng Road, Haidian District, Beijing 100142, China. \\ Email: zhangxiaodong829@163.com.
}

Background: Tumor markers play an important role in the diagnosis, monitoring and prognostic prediction of cancers. But the predictive value of serum tumor markers in gastric cancer is still unclear.

Methods: In this study, we detected serum levels of tumor markers to evaluate their relation to treatment response and prognosis in patients with unresectable advanced or metastatic gastric cancer.

Results: We collected the clinical data of 109 patients with unresectable advanced or metastatic gastric cancer who had received the first-line chemotherapy in Peking University Cancer Hospital from July 2013 to May 2015, and collected the value of serum carcinoembryonic antigen (CEA), carbohydrate antigen 199 (CA199), carbohydrate antigen 72.4 (CA72.4) and carbohydrate antigen 125 (CA125) before and after chemotherapy. At diagnosis, the positive rates of CEA, CA199, CA72.4 and CA125 were 46.8\%, 40.2\%, 53.5\% and $35.0 \%$, respectively. And the positive rate of combined detection of the four markers was $87.2 \%$. Although patients with prechemotherapy CA199 $\geq 80 \mathrm{U} / \mathrm{mL}(92.3 \%$ vs. $68.5 \%, \mathrm{P}=0.016)$ or CA72.4 $\geq 20 \mathrm{U} / \mathrm{mL}(91.4 \%$ vs. $62.5 \%, \mathrm{P}=0.003)$ had higher clinical benefit rate after chemotherapy, they showed poorer prognosis $(\mathrm{P}=0.023$ and $\mathrm{P}=0.006$, respectively). CA72.4 $\geq 20 \mathrm{U} / \mathrm{mL}$ was an independent unfavorable prognostic factor (Hazard Ratio 4.84; $95 \%$ confidence interval: $1.910-12.262$; $\mathrm{P}=0.001$ ). In patients with increased levels of tumor markers before treatment, the levels of tumor markers decreased after chemotherapy, especially in those with clinical benefit (CEA, CA72.4 reached statistical significance, $\mathrm{P}=0.013$ and $\mathrm{P}=0.029$, respectively). A decrease of CEA $\geq 35 \%$, CA199 $\geq 30 \%$, or CA72.4 $\geq 40 \%$ after chemotherapy had positive prediction value for the response to chemotherapy $(\mathrm{P}=0.016, \mathrm{P}=0.029$, and $\mathrm{P}=0.008$, respectively).

Conclusions: The results showed that both high pre-chemotherapy serum levels of tumor markers (CA199 $\geq 80 \mathrm{U} / \mathrm{mL}$ or CA72.4 $\geq 20 \mathrm{U} / \mathrm{mL}$ ) and a substantial decrease in tumor markers after chemotherapy (CEA $\geq 35 \%$, CA199 $\geq 30 \%$, or CA72.4 $\geq 40 \%$ ) could predict a higher clinical benefit rate in patients with unresectable advanced or metastatic gastric cancer. However, this advantage in short-term response to chemotherapy failed to convert into prolonged survival benefits.

Keywords: Gastric cancer; tumor marker; chemotherapy

Submitted Jun 22, 2019. Accepted for publication Oct 10, 2019.

doi: $10.21037 /$ tcr.2019.10.27

View this article at: http://dx.doi.org/10.21037/tcr.2019.10.27 


\section{Introduction}

Gastric cancer is one of the most common malignancies in the world. Despite a major decline in the incidence and mortality over several decades, gastric cancer is still the fourth most common cause of cancer death in the world (1). China is one of the countries with the highest incidence of gastric cancer, which accounts for $42 \%$ of all new gastric cancer cases in the world (2). The diagnostic rate of early gastric cancer is low, and most patients have lost the chance for receiving radical resection at the time of diagnosis because of the late stage $(3,4)$. For the unresectable locally advanced or metastatic gastric cancer, chemotherapy was the backbone of palliative treatment. The accurate staging, chemotherapy efficacy assessment and prognostic prediction during the whole process are crucial for refining the individualized treatment plan.

Today, medical imaging techniques, for example, includes ultrasound, computed tomography (CT), and magnetic resonance imaging (MRI) are commonly used for staging and efficacy assessment in gastric cancer $(5,6)$. However, these techniques are expensive, time consuming and could increase radiation exposure. Furthermore, lesions such as diffuse peritoneal dissemination, are not detectable using medical imaging techniques. For these reasons it is necessary to find other accurate, convenient and rapid methods to assess chemotherapeutic efficacy.

Tumor markers are groups of active substances secreted by tumor cells or normal cells in the process of carcinogenesis, development, invasion and metastasis, which are usually found in the blood, urine, stool or tissue (7-9). With the development of molecular biology, tumor markers are playing increasingly important role in the screening, diagnosis, monitoring and prognostic prediction of cancers. Some studies showed that tumor markers can be predictors of response to chemotherapy in colorectal cancer, ovarian cancer, breast cancer, pancreatic cancer and so on $(10,11)$, but the predictive value of serum tumor markers in gastric cancer is still unclear $(12,13)$.

In this study, we retrospectively analyzed the serum levels of carcinoembryonic antigen (CEA), carbohydrate antigen 199 (CA199), carbohydrate antigen 72.4 (CA72.4) and carbohydrate antigen 125 (CA125) before and after chemotherapy in 109 patients with unresectable advanced or metastatic gastric cancer who had received the first-line chemotherapy in Peking University Cancer Hospital. And we analyzed the value of these four tumor markers in diagnosis, assessment of chemotherapy efficacy and prognostic prediction.

\section{Methods}

\section{Patients}

The medical records of 109 patients with unresectable advanced or metastatic gastric cancer who had received the first-line chemotherapy at the VIP-II Gastrointestinal Cancer Division of Medical Oncology Department of Peking University Cancer Hospital between July 2013 and May 2015 were retrospectively reviewed. The patient inclusion criteria were as follows: (I) pathological diagnosis of gastric cancer; (II) unresectable advanced or metastatic gastric cancer; (III) use of the first-line chemotherapy; (IV) Eastern Cooperative Oncology Group (ECOG) score 0-2 points; (V) expected survival time $\geq 3$ months; (VI) availability of follow-up data. The end of follow-up was March 2016. The study was approved by Peking University Cancer Hospital Research Ethics Committee (2018KT95) and informed consent was obtained from all patients.

\section{The first-line chemotherapy}

All 109 patients received the first-line chemotherapy. Sixty patients $(55.0 \%)$ received platinum-based chemotherapy, and 44 patients $(40.4 \%)$ received taxol-based chemotherapy. Five patients used other chemotherapy regiments. The median number of cycles administered was four (range: one to ten cycles).

\section{Evaluation of clinical response}

The Response Evaluation Criteria in Solid Tumors Revision 1.1 (RECIST 1.1) was used to evaluate the efficacy. Responses were divided into the complete response (CR), partial response (PR), stable disease (SD) or progressive disease (PD). Patients with SD were divided into two groups: shrunken SD (tumor reduction $<30 \%$ ) and enlarged SD (tumor increase $<20 \%$ ). Then further divided into two groups according to the response to chemotherapy: clinical benefit group (including patients with CR, PR, and shrunken SD) and non-clinical benefit group (including patients with PD and enlarged SD). Clinical benefit rate (CBR) was calculated as follows: $(\mathrm{CR}+\mathrm{PR}+$ shrunken $\mathrm{SD}) /$ total patients.

\section{Serum assays for CEA, CA19-9, CA72-4, and CA125}

CEA, CA19-9, CA72.4 and CA125 were assayed by electrochemiluminescence immunoassay (E170, Roche Diagnostics, Switzerland). The cut-off values for serum CEA, 
Table 1 Patient characteristics at baseline $(n=109)$

\begin{tabular}{|c|c|c|}
\hline Characteristics & No. of patients & $\%$ \\
\hline \multicolumn{3}{|l|}{ Gender } \\
\hline Male & 90 & 82.6 \\
\hline Female & 19 & 17.4 \\
\hline \multicolumn{3}{|l|}{ Age, years } \\
\hline$\leq 70$ & 99 & 90.8 \\
\hline$>70$ & 10 & 9.2 \\
\hline \multicolumn{3}{|l|}{ Lauren classification } \\
\hline Intestinal & 45 & 41.3 \\
\hline Diffuse & 34 & 31.2 \\
\hline Mixed & 16 & 14.7 \\
\hline Not known & 14 & 12.8 \\
\hline \multicolumn{3}{|l|}{ Grade } \\
\hline Well-moderate differentiated & 29 & 26.6 \\
\hline Poorly differentiated & 72 & 66.1 \\
\hline Not known & 8 & 7.3 \\
\hline \multicolumn{3}{|l|}{ Disease status } \\
\hline Locally advanced & 26 & 23.9 \\
\hline Metastatic & 83 & 76.1 \\
\hline \multicolumn{3}{|l|}{ Chemotherapy regimens } \\
\hline Platinum-based & 60 & 55.0 \\
\hline Taxol-based & 44 & 40.4 \\
\hline Other & 5 & 4.6 \\
\hline \multicolumn{3}{|l|}{ Metastasis sites } \\
\hline Liver & 33 & 30.3 \\
\hline Peritoneum & 22 & 20.2 \\
\hline Lung & 12 & 11.0 \\
\hline Ovary & 5 & 26.3 \\
\hline
\end{tabular}

CA19-9, CA72.4 and CA125 were $5.0 \mathrm{ng} / \mathrm{mL}, 37.0 \mathrm{U} / \mathrm{mL}$, 6.7 $\mathrm{U} / \mathrm{mL}$ and $35.0 \mathrm{U} / \mathrm{mL}$, respectively, according to the manufacturer's instructions. A result was considered positive when the marker serum level was higher than the cut-off value. Positive combined detection for two or more markers was defined as at least one marker was higher than its cut-off value.

\section{Statistical analysis}

SPSS 16.0 for Windows (SPSS Inc., Chicago, United
States) was used for statistical analysis. Receiver-operating characteristics (ROC) curve was used to evaluate the ability of tumor markers to predict the response to the firstline chemotherapy. Correlations were assessed using the Spearman rank order correlations. Progression-free survival (PFS) and overall survival (OS) were calculated from the first day of chemotherapy to disease progression and death from any cause, respectively. Univariate survival analysis was performed using the Kaplan-Meier method. The independent prognostic factors for patients were assessed by Cox proportional hazards regression model. T-test was applied to determine the differences between mean levels of tumor markers before and after the first-line chemotherapy. $\mathrm{P}<0.05$ was considered statistically significant.

\section{Results}

\section{Patient characteristics}

The characteristics of 109 patients are presented in Table 1 . The median age of enrolled patients was 58.0 years (range, 25-77 years). There were 90 males and 19 females. 83 patients $(76.1 \%)$ had distant metastasis. Lauren classification of gastric cancer in our patients detected 45 intestinal, 34 diffuse types and 16 mixed types.

\section{Prechemotherapy positivity rates of tumor markers}

The prechemotherapy positivity rates of tumor markers are presented in Table 2. The positivity rates of CEA, CA199, CA72.4 and CA125 were 46.8\%, 40.2\%, 53.5\% and 35.0\%, respectively. When combining two items, the positive rate was $65-77 \%$, in which CA199 + CA72.4 group and CA199 + CA125 group had the highest positive rate. When combining three items, the positive rate was $76-82 \%$, CEA + CA199 + CA125 group had the highest positive rate. The positive rate of the four combined markers was $87.2 \%$.

\section{Association of prechemotherapy tumor markers with response to the first-line chemotherapy}

All 109 patients received the first-line chemotherapy and clinical response to chemotherapy was noted in 93 patients; CR and PR were achieved in $18(19.4 \%)$, shrunken SD in $51(54.8 \%)$, enlarged SD in $16(17.2 \%)$ and PD in $8(8.6 \%)$ of patients. The clinical benefit rate (CBR) was $74.2 \%$. As shown in Table 3, patients with elevated CEA, CA199, CA72.4 and CA125 had higher CBR compared to those 
Table 2 Prechemotherapy positivity rates of tumor markers

\begin{tabular}{lc}
\hline Tumor marker & Positivity rate (\%) \\
\hline CEA & 46.8 \\
CA199 & 40.2 \\
CA72.4 & 53.5 \\
CA125 & 35.0 \\
CEA + CA199 & 65.6 \\
CEA + 72.4 & 68.5 \\
CEA + CA125 & 67.0 \\
CA199 + CA72.4 & 76.5 \\
CA199 + CA125 & 76.5 \\
CA72.4 + CA125 & 72.0 \\
CEA + CA199 + CA72.4 & 76.4 \\
CEA + CA199 + CA125 & 82.0 \\
CEA + CA72.4 + CA125 & 81.0 \\
CA199 + CA72.4 + CA125 & 81.5 \\
CEA + CA199 + CA72.4 + CA125 & 87.2 \\
\hline
\end{tabular}

Table 3 Association of prechemotherapy tumor markers with response to chemotherapy

\begin{tabular}{|c|c|c|c|c|}
\hline \multirow{2}{*}{$\begin{array}{l}\text { Tumor } \\
\text { marker }\end{array}$} & \multirow{2}{*}{$\mathrm{N}$} & \multicolumn{2}{|c|}{ Response [n (\%)] } & \multirow{2}{*}{$P$ value } \\
\hline & & Clinical benefit & Non-clinical benefit & \\
\hline CEA & & & & 0.415 \\
\hline- & 43 & $32(74.4)$ & $11(25.6)$ & \\
\hline+ & 38 & $31(78.9)$ & 7 (21.1) & \\
\hline CA199 & & & & 0.027 \\
\hline- & 46 & $31(67.4)$ & $15(32.6)$ & \\
\hline+ & 34 & $30(88.2)$ & $4(11.8)$ & \\
\hline CA72.4 & & & & 0.195 \\
\hline- & 33 & $23(69.7)$ & $10(30.3)$ & \\
\hline+ & 42 & $34(81.0)$ & $8(19.0)$ & \\
\hline CA125 & & & & 0.428 \\
\hline- & 44 & $32(72.7)$ & $12(27.3)$ & \\
\hline+ & 27 & $21(77.8)$ & $6(22.2)$ & \\
\hline
\end{tabular}

with normal levels, of which CA199 reached statistical significance $(\mathrm{P}=0.027)$. Patients with clinical benefit showed significantly higher pretreatment levels of four tumor markers than patients without clinical benefit, among which CA199, CA72.4 reached statistical significance ( $\mathrm{P}=0.034$ and $\mathrm{P}=0.004$, respectively) (Table 4).

To assess the ability of pre-chemotherapy tumor markers to predict the response to the first-line chemotherapy, the ROC curve was carried out (Figure 1). The area under the ROC curve of CA199 was 0.658 (95\% CI: 0.529 to $0.788, \mathrm{P}=0.038$ ); the optimal cutoff which simultaneously maximized both the sensitivity and specificity of the test was $80 \mathrm{U} / \mathrm{mL}$. The area under the ROC curve of CA72.4 was 0.664 (95\% CI: 0.545 to $0.782, \mathrm{P}=0.037$ ); the optimal cutoff was $20 \mathrm{U} / \mathrm{mL}$.

Spearman correlation and chi-square analysis were used to confirm the relationship between prechemotherapy tumor markers and response to the first-line chemotherapy. The correlation coefficient between CA199 and clinical response was 0.238 , and the $\mathrm{P}$ value was 0.032 . The correlation coefficient between CA72.4 and clinical response was 0.245 , and the $\mathrm{P}$ value was 0.033 . The chisquare analysis showed that patients with pretreatment CA199 $\geq 80 \mathrm{U} / \mathrm{mL}$ had higher clinical benefit rate (CBR) than those patients with CA199 $<80 \mathrm{U} / \mathrm{mL}(92.3 \%$ vs. $68.5 \%, \mathrm{P}=0.016$ ), and pretreatment CA72.4 $\geq 20 \mathrm{ng} / \mathrm{mL}$ also had a positive prediction value for CBR $(91.4 \% v s .62 .5 \%$, $\mathrm{P}=0.003)$ (Table 5).

\section{Association of CA199, CA72.4 with PFS and OS}

In a univariate analysis, the PFS (5.9 vs. 7.7 months, $\mathrm{P}=0.013)$ and $\mathrm{OS}(8.4$ vs. 15.0 months, $\mathrm{P}=0.023)$ was lower in patients with CA199 $\geq 80 \mathrm{U} / \mathrm{mL}$ compared to those with CA199 $<80 \mathrm{U} / \mathrm{mL}$. And the PFS (6.5 vs. 6.8 months, $\mathrm{P}=0.077)$ and $\mathrm{OS}(9.9$ vs. 16.3 months, $\mathrm{P}=0.006)$ was also lower in patients with $\mathrm{CA} 72.4 \geq 20 \mathrm{U} / \mathrm{mL}$ compared to those with CA72.4 $<20 \mathrm{U} / \mathrm{mL}$ (Figure 2). In a multivariate analysis, CA72.4 $\geq 20 \mathrm{U} / \mathrm{mL}$ was an independent prognostic factor (HR 4.84; 95\% CI: 1.910-12.262; $\mathrm{P}=0.001$ ) (Table 6).

\section{Changes of tumor markers and their correlation with response to the first-line chemotherapy}

In the patients with high levels of pretreatment tumor markers, the levels of tumor markers decreased after chemotherapy, CEA and CA72.4 in particular $(\mathrm{P}=0.013$ and $\mathrm{P}=0.029$, respectively) (Table 7). Additionally, both CEA and CA72.4 significantly decreased in the patients with clinical benefit when compared with other tumor markers (Table 8).

To assess the ability of the changes of tumor markers 
Table 4 Association of prechemotherapy levels of tumor markers with response to chemotherapy

\begin{tabular}{lcccc}
\hline Response & CEA $(\mathrm{ng} / \mathrm{mL})$ & CA199 $(\mathrm{U} / \mathrm{mL})$ & $\mathrm{CA} 72.4(\mathrm{U} / \mathrm{mL})$ & $\mathrm{CA125}(\mathrm{U} / \mathrm{mL})$ \\
\hline Clinical benefit & $101.4 \pm 298.4$ & $1,889.1 \pm 6,622.4$ & $151.0 \pm 361.4$ & $122.4 \pm 465.1$ \\
Non-clinical benefit & $22.61 \pm 56.1$ & $50.3 \pm 102.7$ & $8.1 \pm 7.0$ & $37.8 \pm 46.2$ \\
$P$ value & 0.258 & 0.034 & 0.004 & 0.446 \\
\hline
\end{tabular}

A

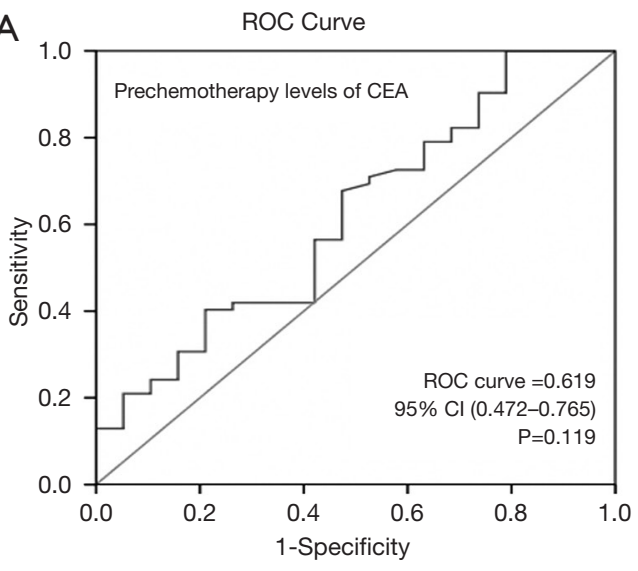

C

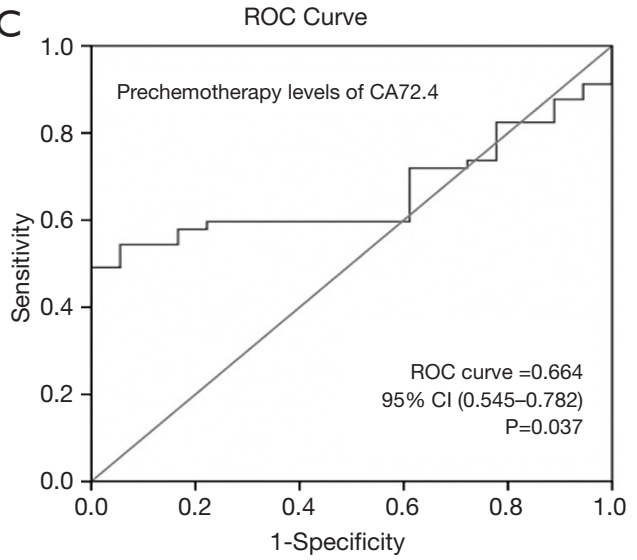

B

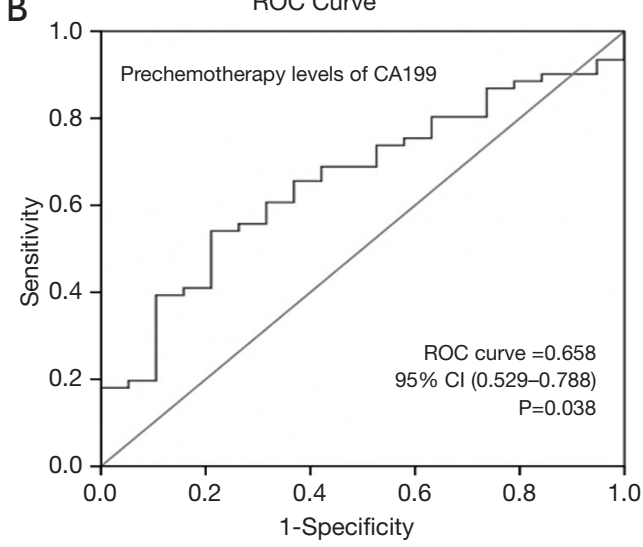

D

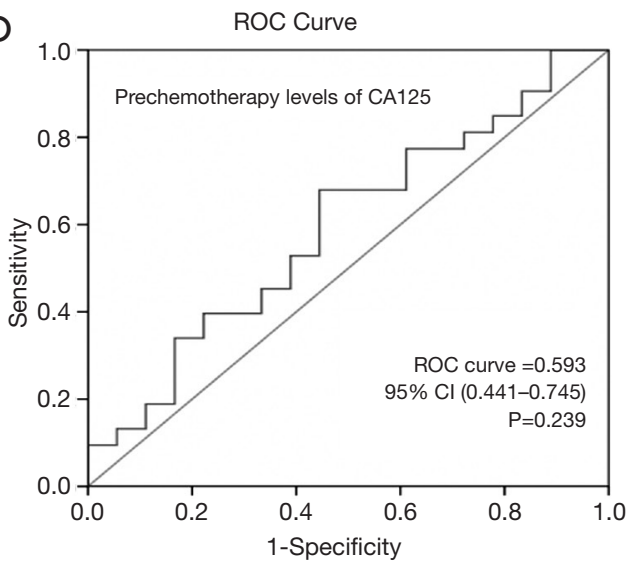

Figure 1 ROC curve for prechemotherapy levels of tumor markers to predict the response to chemotherapy. (B) The area under the ROC curve of CA199 prechemotherapy levels was 0.658 ( $95 \%$ CI: 0.529 to $0.788, \mathrm{P}=0.038$ ); the cutoff that maximized both sensitivity and specificity of this test was $80 \mathrm{ng} / \mathrm{mL}$. (C) The area under the ROC curve of CA72.4 prechemotherapy levels was 0.664 (95\% CI: 0.545 to $0.782, \mathrm{P}=0.037$ ); the cutoff that maximized both sensitivity and specificity of this test was $20 \mathrm{ng} / \mathrm{mL}$. (A,D) Figures show the prechemotherapy levels of CEA and CA125 were not statistically significant (P values were 0.119 , and 0.239 respectively).

after chemotherapy in the prediction of the response to the first-line chemotherapy, the ROC curve was also plotted (Figure 3). The area under the ROC curve of the CEA change value was 0.796 (95\% CI: 0.632 to $0.961, \mathrm{P}=0.025$ ); the optimal cutoff which simultaneously maximized both the sensitivity and specificity of the test was $35 \%$. The area under the ROC curve of CA199 change value was 0.734
(95\% CI: 0.603 to $0.866, \mathrm{P}=0.005$ ) with the optimal cutoff $30 \%$. The area under the ROC curve of CA72.4 and CA125 change value was 0.833 (95\% CI: 0.683 to $0.984, \mathrm{P}=0.011$ ) and 0.725 (95\% CI: 0.559 to $0.891, \mathrm{P}=0.011$ ), with cutoff $40 \%$ and $10 \%$, respectively.

Spearman correlation and chi-square analysis were used to confirm the relationship between tumor markers 
decrease and response to the first-line chemotherapy. The correlation coefficient between CEA decrease, CA199 decrease, CA72.4 decrease, CA125 decrease and clinical response was $0.309(\mathrm{P}=0.008), 0.344(\mathrm{P}=0.004), 0.289$

Table 5 Association of prechemotherapy CA199, CA72.4 with response to chemotherapy

\begin{tabular}{lcccc}
\hline $\begin{array}{l}\text { Tumor } \\
\text { marker }\end{array}$ & $\mathrm{N}$ & \multicolumn{2}{c}{ Response $[\mathrm{n}(\%)]$} & \\
\cline { 5 - 5 } & & Clinical benefit & Non-clinical benefit & \\
\hline CA199 value & & & \\
$\geq 80 \mathrm{U} / \mathrm{mL}$ & 26 & $24(92.3)$ & $2(7.7)$ & \\
$<80 \mathrm{U} / \mathrm{mL}$ & 54 & $37(68.5)$ & $17(31.5)$ & \\
$\mathrm{CA72.4}$ & & & & \\
$\geq 20 \mathrm{U} / \mathrm{mL}$ & 35 & $32(91.4)$ & $3(8.6)$ & \\
$<20 \mathrm{U} / \mathrm{mL}$ & 40 & $25(62.5)$ & $15(37.5)$ & \\
\hline
\end{tabular}
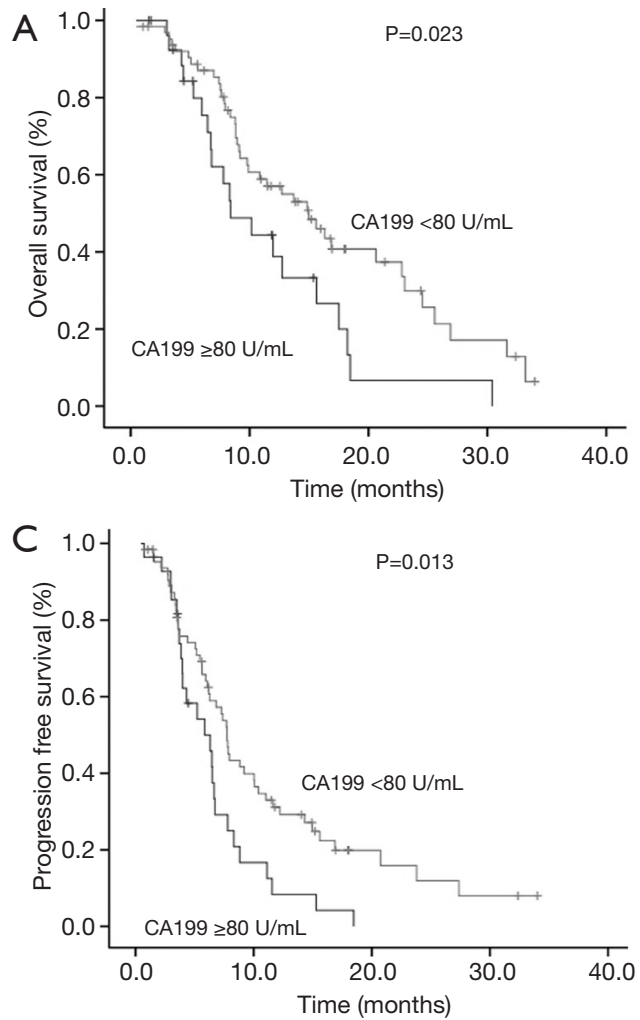

Figure 2 Survival curves of patients according to prechemotherapy level of CA199 and CA72.4. (A) The OS of patients was lower in those with CA199 $\geq 80 \mathrm{U} / \mathrm{mL}$ compared to those with CA199 $<80 \mathrm{U} / \mathrm{mL}$ (8.4 vs. 15.0 months, $\mathrm{P}=0.023$ ). (B) The OS of patients was also lower in those with CA72.4 $\geq 20 \mathrm{U} / \mathrm{mL}$ compared to those with CA72.4<20 U/mL (9.9 vs. 16.3 months, $\mathrm{P}=0.006$ ). (C) The PFS of patients was lower in those with CA199 $\geq 80 \mathrm{U} / \mathrm{mL}$ compared to those with CA199 <80 U/mL (5.9 vs. 7.7 months, $\mathrm{P}=0.013$ ). (D) The PFS of patients was also lower in those with CA72.4 $\geq 20 \mathrm{U} / \mathrm{mL}$ compared to those with CA72.4 $<20 \mathrm{U} / \mathrm{mL}$ (6.5 vs. 6.8 months, $\mathrm{P}=0.077$ ).
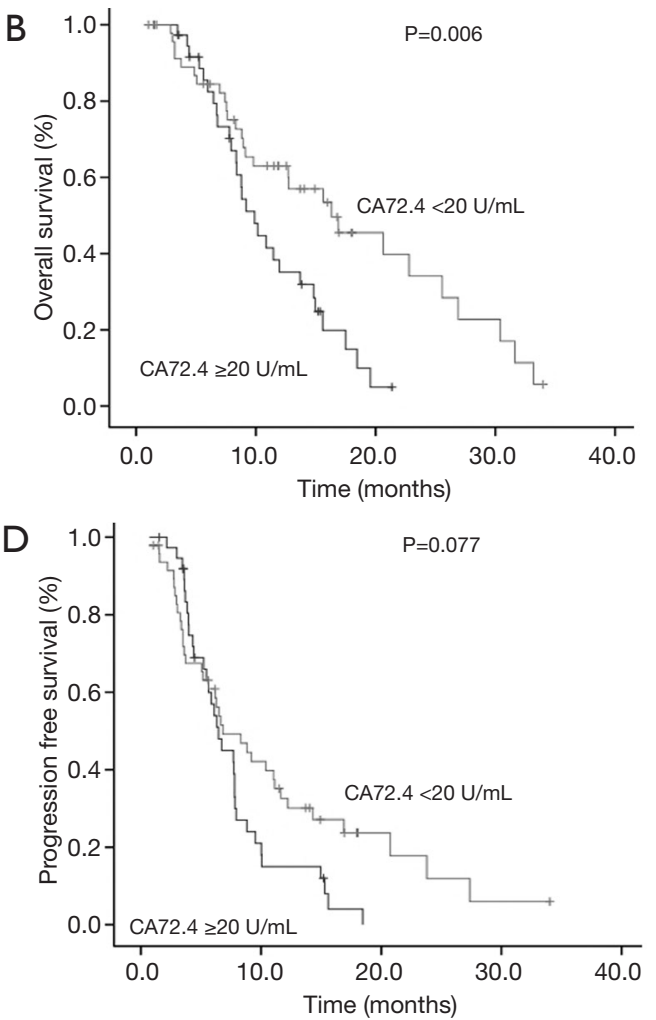

$(\mathrm{P}=0.019)$ and $0.330(\mathrm{P}=0.010)$, respectively. The chi-square analysis showed that a decrease in CEA by more than $35 \%$, CA199 by more than $30 \%$, CA72.4 by more than $40 \%$, had positive predictive value for response to chemotherapy ( $\mathrm{P}=0.016, \mathrm{P}=0.029$, and $\mathrm{P}=0.008$, respectively) (Table 9).

\section{Discussion}

In China, the most commonly used tumor markers of gastric cancer were CEA, CA199, CA72.4 and CA125. In the present study, we analyzed the serum levels of the four tumor markers in 109 patients with unresectable advanced or metastatic gastric cancer before and after the first-line level of tumor markers predicted a higher CBR compared to those with normal levels. And the decrease of tumor markers after chemotherapy was also correlated with treatment response. However, in long-term, both the PFS chemotherapy. In short term, the elevated pretreatment 

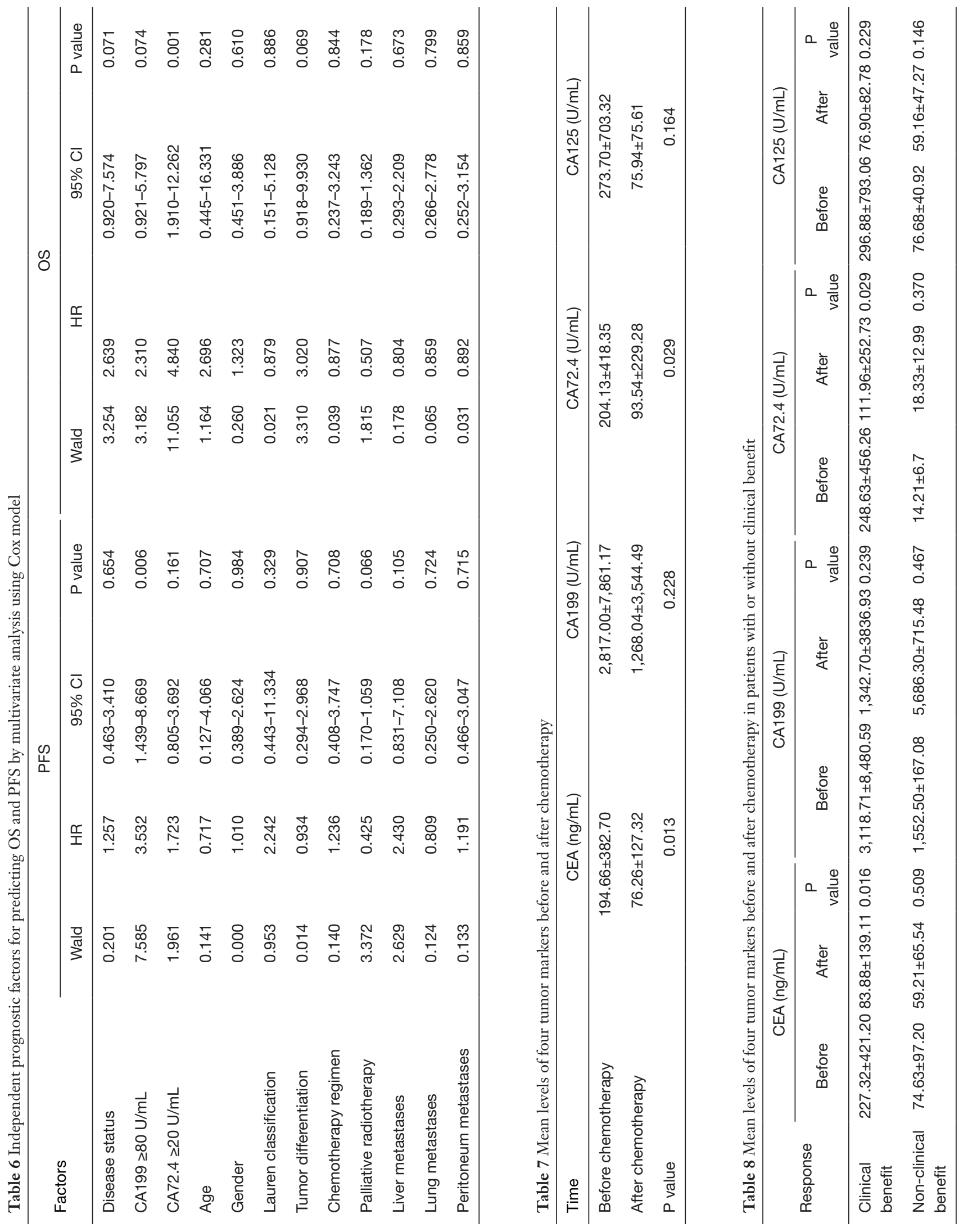

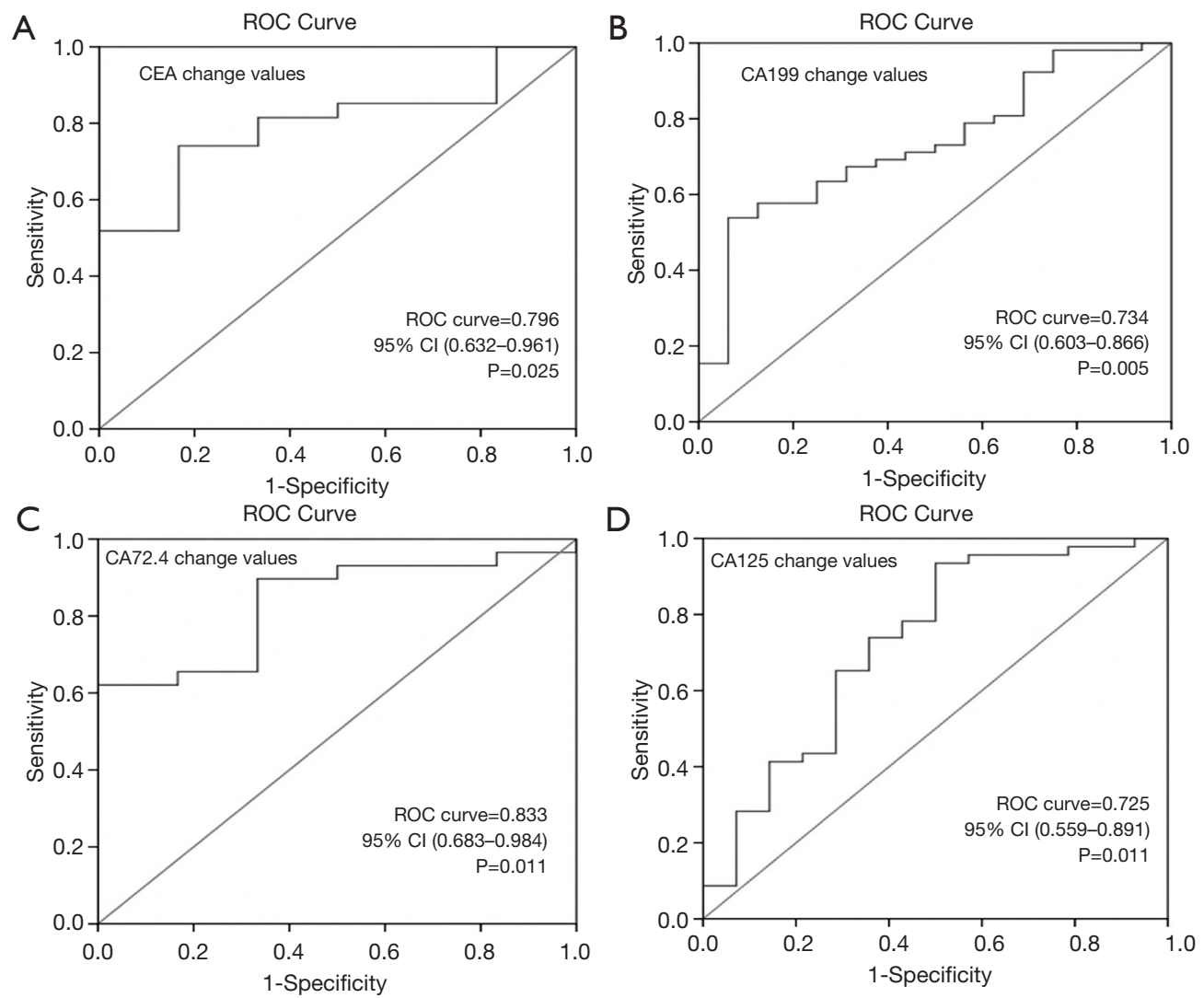

Figure 3 ROC curves for the decrease of the four tumor markers to predict the disease control after the first-line chemotherapy. (A) The area under the ROC curve of CEA change values was 0.796 (95\% CI: 0.632 to $0.961, \mathrm{P}=0.025$ ); the optimal cutoff which simultaneously maximized both the sensitivity and specificity of the test was decreased by $35 \%$. (B) The area under the ROC curve of CA199 change values was 0.734 (95\% CI: 0.603 to $0.866, \mathrm{P}=0.005$ ); the optimal cutoff was decreased by $30 \%$. (C) The area under the ROC curve of CA72.4 change values was 0.833 (95\% CI: 0.683 to $0.984, \mathrm{P}=0.011$ ); the optimal cutoff was decreased by $40 \%$. (D) The area under the ROC curve of CA125 change values was 0.725 (95\% CI: 0.559 to $0.891, \mathrm{P}=0.011$ ); the optimal cutoff was decreased by $10 \%$.

and OS were significantly short in those patients with higher tumor markers before chemotherapy. Besides, CA72.4 $\geq 20 \mathrm{U} / \mathrm{mL}$ was identified as an independent unfavorable prognostic factor.

The reported positive rate of these four tumor markers are varying, depending on the sample size and centers (1420). One review including 46 studies performed by Hideaki Shimada (14) showed that the overall positive rates for CEA, CA199 and CA72.4 were 24.0\%, 27.0\% and 29.9\% respectively, and these three markers were significantly associated with tumor stage. The stage of cancer strongly correlated with the positive rates of tumor markers, in which the later the stage, the higher positive rates. The positive rates for CEA, CA199 and CA72.4 in patients of stage I disease were $13.7 \%, 9.0 \%$ and $12.0 \%$, while positive rates in stage IV patients were $39.5 \%, 44.7 \%$ and $49.6 \%$, respectively. In this study, we analyzed the serum levels of tumor markers in 109 patients with unresectable advanced or metastatic gastric cancer. Our results showed that the positive rates for CEA, CA199, CA72.4 and CA125 were $46.8 \%, 40.2 \%, 53.5 \%$ and $35 \%$, respectively, which was consistent with previous studies $(14,21,22)$. Moreover, the positive rates for combined detection of two, three and four tumor markers were $65-77 \%, 76-82 \%$, and $87.2 \%$, respectively, indicating that the combined evaluation could increase the diagnostic sensitivity.

Previous studies showed that tumor markers were related to response to chemotherapy in many tumors $(10,11,23-25)$. However, the predictive value of serum tumor markers in late-stage gastric cancer is still unclear $(12,13)$. In this study, we found that patients with elevated tumor markers had higher CBR compared to those with normal levels by 
Table 9 Correlation between decrease of tumor markers and clinical response

\begin{tabular}{|c|c|c|c|c|}
\hline \multirow{2}{*}{ Tumor marker } & \multirow{2}{*}{$\mathrm{N}$} & \multicolumn{2}{|c|}{ Response [n (\%)] } & \multirow{2}{*}{$P$ value } \\
\hline & & Clinical benefit & Non-clinical benefit & \\
\hline \multicolumn{4}{|c|}{ Decrease of CEA } & 0.003 \\
\hline$\geq 35 \%$ & 21 & $20(95.2)$ & $1(4.8)$ & \\
\hline$<35 \%$ & 12 & 7 (58.3) & $5(41.7)$ & \\
\hline \multicolumn{4}{|c|}{ Decrease of CA199 } & 0.029 \\
\hline$\geq 30 \%$ & 18 & $18(100.0)$ & $0(0.0)$ & \\
\hline$<30 \%$ & 9 & $6(66.7)$ & 3 (33.3) & \\
\hline \multicolumn{4}{|c|}{ Decrease of CA72.4 } & 0.008 \\
\hline$\geq 40 \%$ & 18 & $18(100.0)$ & $0(0.0)$ & \\
\hline$<40 \%$ & 17 & $11(64.7)$ & $6(35.3)$ & \\
\hline \multicolumn{4}{|c|}{ Decrease of CA125 } & 0.292 \\
\hline$\geq 10 \%$ & 16 & $14(87.5)$ & $2(12.5)$ & \\
\hline$<10 \%$ & 6 & $4(66.7)$ & 2 (33.3) & \\
\hline
\end{tabular}

using chi-square analysis, and CA199 reached statistical significance. We also found that patients with clinical benefit had significantly higher pretreatment levels of CA199 and CA72.4 than patients without clinical benefit. Therefore, we speculated that prechemotherapy serum tumor markers could predict the response to chemotherapy. In order to confirm this presumption, we plotted a ROC curve and found that the pre-chemotherapy level of CA199 and CA72.4 could be used to predict response to chemotherapy; the cut-off value was 80 and $20 \mathrm{U} / \mathrm{mL}$, respectively. Further chi-square analysis showed that patients with pretreatment CA199 $\geq 80 \mathrm{U} / \mathrm{mL}$ had higher CBR than those patients with $\mathrm{CA} 199<80 \mathrm{U} / \mathrm{mL}$ (92.3\% vs. $68.5 \%, \mathrm{P}=0.016)$. And pretreatment CA72.4 $\geq 20 \mathrm{ng} / \mathrm{mL}$ also had higher CBR than those patients CA72.4 $<20 \mathrm{U} / \mathrm{mL}(91.4 \%$ vs. $62.5 \%$, $\mathrm{P}=0.003)$. These results suggested that the serum tumor markers could be used as predictive biomarker before the first-line chemotherapy. This phenomenon was consistent with the previous study in colorectal cancer (26).

Furthermore, the decrease of tumor markers after chemotherapy was also correlated with treatment response in this study. The mean levels of CEA, CA199, CA72.4 and CA125 were decreased after chemotherapy, especially in patients with clinical benefit. The ROC curve showed the decrease of CEA, CA199 and CA72.4 after chemotherapy could predict CBR and the cutoff value decrease by $35 \%$,
$30 \%$ and $40 \%$, respectively. Further chi-square analysis showed that patients with CEA decrease $\geq 35 \%$, CA199 decrease $\geq 30 \%$ and CA72.4 decrease $\geq 40 \%$ had higher CBR than the control group $(\mathrm{P}=0.016, \mathrm{P}=0.029$ and $\mathrm{P}=0.008$, respectively). This finding was in agreement with our knowledge that the level of tumor marker could reflect tumor burden, and the change of tumor marker level reflects the change of tumor burden $(13,27)$. One previous study reported that a decrease (>70\%) of CA72-4 may predict pathologic response to neoadjuvant chemotherapy in gastric cancer (12). Based on the result, we think that tumor markers might be useful in monitoring the chemotherapy response in gastric cancer, especially for those cases in which disease is difficult to evaluate by imaging, such as diffuse peritoneal dissemination.

Although high level of prechemotherapy tumor marker predicted better response to chemotherapy, the advantage does not associate with a survival benefit. Both the PFS and OS were significantly short in those patients with high tumor markers before treatment. Multivariate analysis also identified CA72.4 $\geq 20 \mathrm{U} / \mathrm{mL}$ was an independent unfavorable prognostic factor (HR 4.84; $95 \%$ CI: 1.910 12.262; $\mathrm{P}=0.001)$. The results were in line with previous studies, which showed that high levels of preoperative CEA, CA199, and CA72.4 may be associated with poor prognosis in patients with resected gastric cancer (28-34).

The results of this study had some significance for clinical practice. Prognosis of patients with a high level of tumor markers are poor, but they had better short-term response to chemotherapy, which brings opportunities to take local treatments. Therefore, we should pay close attention to the evaluation of the response to chemotherapy, in order to grasp the opportunity of local treatments and bring benefits to these patients to the greatest degree.

The limitations of our study include that the study was retrospective and the number of patients was small. Moreover, not all patients were tested for all four tumor markers, so there was loss of some data. The results should be further validated in large-scale prospective studies with complete patient data.

\section{Conclusions}

In conclusion, tumor markers have clinical value in the diagnosis of disease, chemotherapy efficacy assessment and prognostic prediction in patients with unresectable advanced or metastatic gastric cancer. Patients with prechemotherapy CA199 $\geq 80 \mathrm{U} / \mathrm{mL}$ or CA72.4 $\geq 20 \mathrm{U} / \mathrm{mL}$ 
had a better short-term response to chemotherapy, but this advantage failed to convert into long-term survival benefits. On the contrary, these patients had poorer prognosis. A decrease $\geq 35 \%$ of CEA, decrease $\geq 30 \%$ of CA199 and decrease $\geq 40 \%$ of CA72.4 may predict clinical benefit after chemotherapy. These results may provide help for the individualized treatment of patients with gastric cancer.

\section{Acknowledgments}

Funding: None.

\section{Footnote}

Conflicts of Interest: All authors have completed the ICMJE uniform disclosure form (available at http://dx.doi. org/10.21037/tcr.2019.10.27). The authors have no conflicts of interest to declare.

Ethical Statement: The authors are accountable for all aspects of the work in ensuring that questions related to the accuracy or integrity of any part of the work are appropriately investigated and resolved. The study was conducted in accordance with the Declaration of Helsinki (as revised in 2013). The study protocol was approved by the Peking University Cancer Hospital Research Ethics Committee (2018KT95) and informed consent was obtained from all patients.

Open Access Statement: This is an Open Access article distributed in accordance with the Creative Commons Attribution-NonCommercial-NoDerivs 4.0 International License (CC BY-NC-ND 4.0), which permits the noncommercial replication and distribution of the article with the strict proviso that no changes or edits are made and the original work is properly cited (including links to both the formal publication through the relevant DOI and the license). See: https://creativecommons.org/licenses/by-nc-nd/4.0/.

\section{References}

1. Siegel RL, Miller KD, Jemal A. Cancer statistics, 2015. CA Cancer J Clin 2015;65:5-29.

2. Li G, Hu Y, Liu H. Current status of randomized controlled trials for laparoscopic gastric surgery for gastric cancer in China. Asian J Endosc Surg 2015;8:263-7.

3. Macdonald JS. Gastric cancer--new therapeutic options. N Engl J Med 2006;355:76-7.
4. Wang X, Wei Y, Xue Y, et al. Predictive Role of the Number of 18F-FDG-Positive Lymph Nodes Detected by PET/CT for Pre-Treatment Evaluation of Locally Advanced Gastric Cancer. PLoS One 2016;11:e0166836.

5. Therasse P, Arbuck SG, Eisenhauer EA, et al. New guidelines to evaluate the response to treatment in solid tumors. European Organization for Research and Treatment of Cancer, National Cancer Institute of the United States, National Cancer Institute of Canada. J Natl Cancer Inst 2000;92:205-16.

6. Shanbhogue AK, Karnad AB, Prasad SR. Tumor response evaluation in oncology: current update. J Comput Assist Tomogr 2010;34:479-84.

7. Sharma S. Tumor markers in clinical practice: General principles and guidelines. Indian J Med Paediatr Oncol 2009;30:1-8.

8. Perkins GL, Slater ED, Sanders GK, et al. Serum tumor markers. Am Fam Physician 2003;68:1075-82.

9. Gara S, Boussen $\mathrm{H}$, Ghanem A, et al. Use of common seric tumor markers in patients with solid cancers. Tunis Med 2008;86:579-83.

10. Iwanicki-Caron I, Di Fiore F, Roque I, et al. Usefulness of the serum carcinoembryonic antigen kinetic for chemotherapy monitoring in patients with unresectable metastasis of colorectal cancer. J Clin Oncol 2008;26:3681-6.

11. Kang S, Kim TJ, Seo SS, et al. Interaction between preoperative CA-125 level and survival benefit of neoadjuvant chemotherapy in advanced epithelial ovarian cancer. Gynecol Oncol 2011;120:18-22.

12. Sun Z, Zhang N. Clinical evaluation of CEA, CA199, CA72-4 and CA125 in gastric cancer patients with neoadjuvant chemotherapy. World J Surg Oncol 2014;12:397.

13. Yamao T, Kai S, Kazami A, et al. Tumor markers CEA, CA19-9 and CA125 in monitoring of response to systemic chemotherapy in patients with advanced gastric cancer. Jpn J Clin Oncol 1999;29:550-5.

14. Shimada H, Noie T, Ohashi M, et al. Clinical significance of serum tumor markers for gastric cancer: a systematic review of literature by the Task Force of the Japanese Gastric Cancer Association. Gastric Cancer 2014;17:26-33.

15. Gaspar MJ, Arribas I, Coca MC, et al. Prognostic value of carcinoembryonic antigen, CA 19-9 and CA 72-4 in gastric carcinoma. Tumour Biol 2001;22:318-22.

16. Liu X, Qiu H, Liu J, et al. Combined preoperative concentrations of CEA, CA 19-9, and 72-4 for predicting outcomes in patients with gastric cancer after curative 
resection. Oncotarget 2016;7:35446-53.

17. Distler M, Pilarsky E, Kersting S, et al. Preoperative CEA and CA 19-9 are prognostic markers for survival after curative resection for ductal adenocarcinoma of the pancreas - a retrospective tumor marker prognostic study. Int J Surg 2013;11:1067-72.

18. Lee JC, Lee SY, Kim CY, et al. Clinical utility of tumor marker cutoff ratio and a combination scoring system of preoperative carcinoembryonic antigen, carbohydrate antigen 19-9, carbohydrate antigen 72-4 levels in gastric cancer. J Korean Surg Soc 2013;85:283-9.

19. Tian SB, Yu JC, Kang WM, et al. Combined detection of CEA, CA 19-9, CA 242 and CA 50 in the diagnosis and prognosis of resectable gastric cancer. Asian Pac J Cancer Prev 2014;15:6295-300.

20. Reiter W, Stieber P, Reuter C, et al. Prognostic value of preoperative serum levels of CEA, CA 19-9 and CA 72-4 in gastric carcinoma. Anticancer Res 1997;17:2903-6.

21. Yang AP, Liu J, Lei HY, et al. CA72-4 combined with CEA, CA125 and CA19-9 improves the sensitivity for the early diagnosis of gastric cancer. Clin Chim Acta 2014;437:183-6.

22. Kim JH, Jun KH, Jung H, et al. Prognostic Value of Preoperative Serum Levels of Five Tumor Markers (Carcinoembryonic Antigen, CA19-9, Alphafetoprotein, CA72-4, and CA125) in Gastric Cancer. Hepatogastroenterology 2014;61:863-9.

23. Zeng WG, Liang JW, Wang Z, et al. Clinical parameters predicting pathologic complete response following neoadjuvant chemoradiotherapy for rectal cancer. Chin J Cancer 2015;34:468-74.

24. Chudecka-Głaz AM, Cymbaluk-Ploska AA, Menkiszak JL, et al. Serum HE4, CA125, YKL-40, bcl-2, cathepsin-L and prediction optimal debulking surgery, response to chemotherapy in ovarian cancer. J Ovarian Res 2014;7:62.

25. Wang YJ, Huang XY, Mo M, et al. Serum Tumor Marker
Levels might have Little Significance in Evaluating Neoadjuvant Treatment Response in Locally Advanced Breast Cancer. Asian Pac J Cancer Prev 2015;16:4603-8.

26. Sorbye H, Mauer M, Gruenberger T, et al. Predictive factors for the benefit of perioperative FOLFOX for resectable liver metastasis in colorectal cancer patients (EORTC Intergroup Trial 40983). Ann Surg 2012;255:534-9.

27. Căinap C, Nagy V, Gherman A, et al. Classic tumor markers in gastric cancer. Current standards and limitations. Clujul Med 2015;88:111-5.

28. Kochi M, Fujii M, Kanamori N, et al. Evaluation of serum CEA and CA19-9 levels as prognostic factors in patients with gastric cancer. Gastric Cancer 2000;3:177-86.

29. Park SH, Ku KB, Chung HY, et al. Prognostic significance of serum and tissue carcinoembryonic antigen in patients with gastric adenocarcinomas. Cancer Res Treat 2008;40:16-21.

30. Ishigami S, Natsugoe S, Hokita S, et al. Clinical importance of preoperative carcinoembryonic antigen and carbohydrate antigen 19-9 levels in gastric cancer. J Clin Gastroenterol 2001;32:41-4.

31. Liu X, Cai H, Wang Y. Prognostic significance of tumor markers in T4a gastric cancer. World J Surg Oncol 2012;10:68.

32. Liu X, Cai H, Wang Y. Prognostic significance of tumour markers in Chinese patients with gastric cancer. ANZ J Surg 2014;84:448-53.

33. Tachibana M, Takemoto Y, Nakashima Y, et al. Serum carcinoembryonic antigen as a prognostic factor in resectable gastric cancer. J Am Coll Surg 1998;187:64-8.

34. Song YX, Huang XZ, Gao P, et al. Clinicopathologic and Prognostic Value of Serum Carbohydrate Antigen 19-9 in Gastric Cancer: A Meta-Analysis. Dis Markers 2015;2015:549843.
Cite this article as: Sun Z, Jia J, Du F, Yang Y, Liu C, Xiao Y, Yu J, Zhang X. Clinical significance of serum tumor markers for advanced gastric cancer with the first-line chemotherapy. Transl Cancer Res 2019;8(8):2680-2690. doi: 10.21037/tcr.2019.10.27 